\title{
Rheological characterization of two different suspension formulations of beclomethasone dipropionate enemas for rectal administration
}

\author{
Anna Vittoria Demma ${ }^{1 *}$, Franco Sartor ${ }^{2}$, Gian Eugenio Tontini ${ }^{3}$, Luca Pastorelli ${ }^{4}$ and Maurizio Vecchi ${ }^{5}$
}

*Correspondence: annavittoriademma@gmail.com

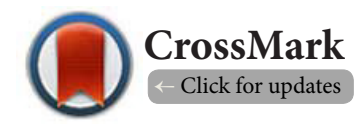

'Philip Morris Manufacturing \&Technology Bologna, Bologna, Italy.

${ }^{2}$ Chiesi Farmaceutici SpA, Parma, Italy.

${ }^{3}$ RCCS Fondazione Ca' Granda, Ospedale Maggiore Policlinico, Milan, Italy.

${ }^{4}$ Gastroenterology and Gastrointestinal Endoscopy Unit, IRCCS Policlinico San Donato, San Donato Milanese (MI), Italy.

IIRCCS Fondazione Ca' Granda, Ospedale Maggiore Policlinico, Milan, Italy; Department of Pathophysiology and Transplantation,

University of Milan, Milan, Italy.

\begin{abstract}
Background: The characterization of the rheological properties of topical formulations is important to predict their possible behaviour in clinical use.

Methods: Two commercially available suspension formulations of beclomethasone dipropionate (BDP) enemas (Formulation A and formulation B) were analysed to define their rheological characteristics under different conditions.

Results: The different composition of the two tested formulations led to important differences in their rheological properties. The results showed that formulation A has viscoelastic gel-like properties, whereas formulation B has viscous-fluid characteristics. A gel-like behaviour and pronounced elastic properties may determine longer time of drug permanence in the site of action. Formulation A retained its rheological properties to a greater extent than did Formulation B across a range of stresses that simulate the agitation of the container before using. A gel-like behaviour may be associated to a high ability to recompose the deformation once the stress is ceased. A more pronounced structural integrity may be indicative of a higher stability of formulation A compared to formulation B.

Conclusions: These different rheological properties between the two examined BDP enemas formulations may lead to important differences in the performance in clinical practice, which should be demonstrated in appropriate randomized clinical trials.
\end{abstract}

Keywords: Beclometasone Diproprionate, suspension, colitis, rheological viscosity

\section{Introduction}

Inflammatory bowel diseases (IBD) are a group of chronic and relapsing inflammatory disorders of the gastrointestinal tract (GI). IBD includes Ulcerative Colitis (UC) and Crohn's Disease (CD). Whilst disease localization to the rectum, sigmoid and/or descending colon occurs in a discrete proportion of CD patients, UC always involves the rectum and may extend proximally up to the caecum; as such, most of IBD patients have the disease involving the left/distal colon [1]. Approximately $80 \%$ of all incident cases of UC are mild or moderate in severity, and more than $75 \%$ of patients have an endoscopic involvement distal to the splenic flexure [2], although proximal extension is not uncommon and should be considered in case of worsening of the clinical conditions. The disease can be limited to the intestine or may affect the skin, joints, spine, liver, eyes, and other organs [3]. Although people of any age can have IBD, the diagnosis is most commonly first made in young adults [4].

Medical therapy for IBD relies on drugs with anti-inflammatory properties. Despite their wide use, treatment with corticosteroids (CS) is associated with known adverse effects [5]. The local administration of CS by means of enema or suppositories allows delivering high drug concentrations directly to the site of inflammation, while avoiding systemic adverse effects. In fact, topical treatment of active distal UC with enemas containing 
glucocorticosteroids is a well established therapy from several decades [6-9]. Moreover, low-bioavailability CS formulations of budesonide and beclometasone dipropionate (BDP) have been introduced in clinical practice. These formulations are characterised by a prompt and potent anti-inflammatory activity and a low systemic bioavailability [10], which is mainly achieved through low intestinal absorption and extensive first-pass metabolism. A very high hepatic clearance following the first hepatic pass is obtained through portal circulation [7]. These newer compounds are widely used for mild to moderate UC or CD due to their safer profile compared to older systemic $\mathrm{CS}$, while maintaining equivalent efficacy $[11,12]$.

From a manufacturing perspective, oral controlled-release formulations of BDP and budesonide have been developed aimed at releasing the active drug directly at the site of action [1]. Other recently oral formulations have been developed to deliver and release high concentrations of active drugs with a homogeneous distribution along all the different colonic segments [13]. In addition to oral forms, CS are also available as rectal suspension enemas or suppositories and their use in clinical practice is well established. Several controlled clinical trials have demonstrated that topical formulations of BDP are beneficial in the treatment of active distal UC, with an efficacy comparable to that of oral CS [14] or even superior to that of aminosalicylates [15]. Moreover, the topical treatment is not associated with suppression of the hypothalamus-pituitary adrenal axis [16].

The pharmaceutical forms used in topical therapy such as ointments, creams and suspensions are frequently object of rheological analysis, since for such products viscosity and sliding properties are closely related to the performance of the drug. In fact, the effectiveness of these products is generally affected by the nonspecific loss of the drug due to the ejection from the patient and by an erratic absorption in the mucosal tissue. Moreover, the target area is often characterized by a modest surface of contact with the drug. A strategy to contain such limitations and disadvantages that characterize suspensions for internal topical use consists in formulating pharmaceutical forms with particular care to the choice of thickeners agents. In fact, it is reasonable to think that a higher viscosity of the suspension, a viscoelastic gel-like behaviour and accentuated elastic properties determine longer times of drug permanence in the desired site of action. This will reduce the ease and frequency of expulsion of the product and increases the time of exposure of the affected surface to the active drug, with a consequent improvement of the compliance (since it will not require additional doses) and, therefore, the therapeutic action of the drug may be maximized.

Furthermore, it should be taken into consideration that marketed products are generally made available in bottles. Thus, a true solicitation of the suspensions takes place during the application, which is often a self-administration by squeezing. The mechanical stress clearly modifies the rheological properties of the material as a consequence of deformation during the flow. This results in more or less marked variations of viscoelastic behaviour and viscosity, and of the consequent consistency of the product. Once the external mechanical stress is ceased, this translates in a different tendency to remain in the target therapeutic area where the therapeutic effect is required.

Based on this background, we have considered of interest to characterize and compare two BDP suspension formulations for mucosal topical use. In particular, the objective of our studies was to define the viscoelastic behaviour and the rheological parameters of the two formulations, and to highlight differences in the consistency of pharmaceutical forms that might correlate with the products' performance in clinical use.

\section{Methods}

Two currently marketed BDP aqueous suspension formulations (formulation A and formulation B) for topical mucosal application have been analyzed. The active substance is dosed at the same concentration in the two formulations.

The composition of the two formulations is as follows:

Formulation A: methyl p-Hydroxybenzoate, propyl p- Hydroxybenzoate, Na Phosphate Dibasic DiHydrate, Na PhosphateMono basic, BenzilicAlchol, Cestostearilic Alcohol, XANTHAN GUM, poly sorbate 20 , Sorbitanmonolaurate, Purified Water.

Formulation B: methyl p-Hydroxybenzoate, Ethyl p- Hydroxybenzoate, EthylenDiaminoTertracetic $\mathrm{Na}$, Na PhosphateMono basic di hydrate, Na Phosphate Dibasic Dodeca-hydrate, Na carboxymethyl cellulose, Purified Water.

As reported above, the composition of the two pharmaceutical products differs for the excipients used as preservatives, $\mathrm{pH}$ modulators, emulsifiers and thickener agents (Xanthan Gum and $\mathrm{NaCMC}$ respectively in the A and B formulation, respectively). Both products are packed in disposable bottles of the same volumetric capacity. Rheological measures were made using a TA rotational rheometer - ARG2 Instrument. Rotational rheometers are among the more suitable tools for measurements on liquid-like samples, such as the aqueous suspension that is the object of this study. These instruments allow performing both flow tests (viscosimetry) and oscillatory tests. The first is based on the relationship between shear stress and shear rate when the sample is subjected to a shear flow, and allows getting viscosity measures, especially for not too consistent or semisolid systems. The second one, the oscillatory or dynamic test, is based on the relationship between the shear stress and the shear rate when the sample is subjected to an oscillatory shear flow with a certain frequency, and is used for the determination of viscoelastic parameters. In addition, they can also apply normal stress, for samples analysed by means of the creep test or the stress relaxation in compression. The instrument is also equipped with a fixed lower Peltier plate on which the sample is deposited or with a device which enables a tight control of the temperature by the operator (temperature range from $-40^{\circ} \mathrm{C}$ to $100^{\circ} \mathrm{C}$ ). 
The plate-cone was the geometry used for the measurements, as it is the most appropriate for samples with limited viscosity and adequate to exercise a real shear stress on the sample. The steel cone has a diameter of $40 \mathrm{~mm}$ and an angle of 0.59 degrees. The most important errors using a measuring system with this geometry are essentially an error in the gap between the cone and plate and the improper filling with the sample of the space between the cone and the plate [17]. The first potential error was avoided by using a geometry of a truncated cone with a fixed Gap at $27 \mu \mathrm{m}$, defined by the manufacturer of the rheometer itself. The second error is due to the operator and, as such, it is not completely removable. Another caution consisted in placing a small amount of water in the upper groove of the cone to prevent the sample evaporation during the analysis.

The rheological tests were performed on two different batches of each suspension-sample. For simplicity hereinafter we will identify the samples tested with "batch $A 1$ " and "batch A2" for formulation A, and similarly with "batch B1" and "batch B2" for formulation B. Each test was made at room temperature $\left(25^{\circ} \mathrm{C}\right)$ and at body temperature $\left(37^{\circ} \mathrm{C}\right)$. Each measure was taken in triplicate and average values were calculated.

The following tests were performed:

1. Oscillation stress sweep. The sample was subjected to an increasing stress amplitude (from $10^{-3} \mathrm{~Pa}$ to $10^{2} \mathrm{~Pa}$ ) at constant Micro-Macro-interaction frequency $(0.5$ $\mathrm{Hz}$ ). The $\mathrm{G}^{\prime}$ obtained values were plotted in logarithmic scale. This test is carried out to define the linear viscoelastic range (LVR) of the samples to assess threshold and Micro-Macro-interaction flow parameters $\left(\sigma_{y}\right.$ and $\sigma_{f}$ ) and to choose the appropriate stress values to be applied in the subsequent tests.

2. Creep Recovery. The sample was subjected for $100 \mathrm{sec}-$ onds to a constant amplitude stress, evaluated on its LVR and $\sigma_{\mathrm{y}}$ values. Instant removal of the stress at $\mathrm{t}=100 \mathrm{sec}$ and then a recovery stage of a duration of 300 seconds followed. The value of percentage deformation $(\gamma \%)$ of the sample was reported as a function of time.

3. Frequency sweep. The sample was subjected to an increasing frequency (from $2.10 \mathrm{rad} / \mathrm{sec}$ to $102 \mathrm{rad} / \mathrm{sec}$ ) at constant amplitude, evaluated according to the $\sigma_{y}$ and LVR of each sample. The frequency range and the $\mathrm{G}^{\prime}$ obtained values were plotted in logarithmic scale.

4. Viscosimetry test. The sample was subjected to a solicitation with increasing shear rate (from $10^{-3} \mathrm{sec}^{-1}$ to $10^{3} \mathrm{sec}^{-1}$ ). Flow and viscosity curves were recorded, and were then fitted with the Rheosoft software through mathematical models of 'best fitting' in order to obtain the characteristic parameters of yield stress, zero rate viscosity and infinity rate viscosity.

5. Time sweep. The sample was subjected to a solicitation of constant stress (based on its LVR and $\sigma_{y}$ ) at constant frequency of $0.5 \mathrm{~Hz}$, in order to assess time dependent changes in $\mathrm{G}^{\prime}$ and $\mathrm{G}^{\prime \prime}$ modules.

6. Temperature sweep. The test was conducted by submitting the sample to a constant stress solicitation (rated according to its LVR and $\sigma_{\mathrm{y}}$ ) at constant frequency of 0.5 $\mathrm{Hz}$, in order to evaluate variations in the modules $\mathrm{G}^{\prime}$ and $\mathrm{G}^{\prime \prime}$ in the range of increasing temperature from $5^{\circ} \mathrm{C}$ to $45^{\circ} \mathrm{C}$ and in the corresponding temperature cooling ramp from $45^{\circ} \mathrm{C}$ to $5^{\circ} \mathrm{C}$ (heat rate $2^{\circ} \mathrm{C} / \mathrm{min}$, cool rate $2^{\circ} \mathrm{C} / \mathrm{min}$ ).

\section{Results}

\section{Oscillation stress sweep}

Figure 1 shows the results of the oscillation stress sweep tests with the two formulations at room $\left(25^{\circ} \mathrm{C}\right)$ and body $\left(37^{\circ} \mathrm{C}\right)$ temperature. Table 1 shows the yield stress and stress flow values of the two tested batches of formulation $A$ and $B$ at room temperature $\left(25^{\circ} \mathrm{C}\right)$ and body temperature $\left(37^{\circ} \mathrm{C}\right)$.

In both batches of formulation $A$, the elastic modulus $G^{\prime}$ was always greater than the viscous modulus $G^{\prime \prime}$ at both room and body temperature. The $\mathrm{G}^{\prime}$ value decreased as temperature increased by $20 \%$, since the sample is an aqueous suspension and at high temperatures such samples increase their viscose fluid component and therefore their tendency to flow. The reproducibility of the measurements intra-batch and inter-batches was very high at $25^{\circ} \mathrm{C}$, whereas at $37^{\circ} \mathrm{C}$ measures remained highly reproducible intra-batch but were less reproducible inter-batches (Table 1 ).

In both batches of formulation $B$, the modulus $G^{\prime \prime}$ was always greater than G'. Also in this case, as temperature increased the

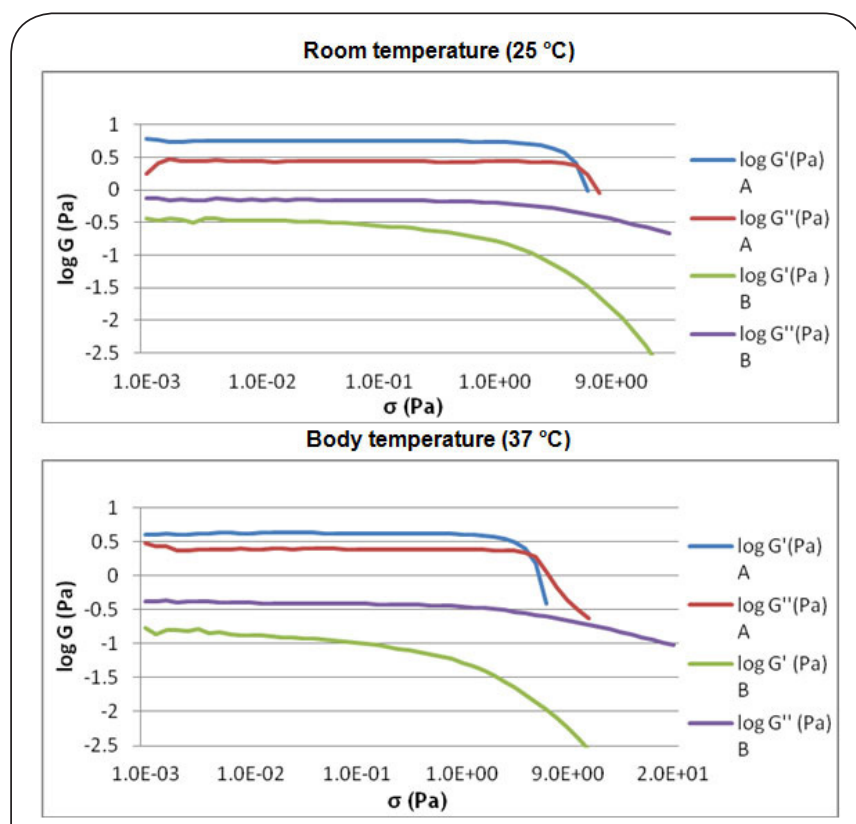

Figure 1. Results of oscillation stress sweep tests. Comparisons of logarithm (log) of the storage ( $\left.G^{\prime}\right)$ and Loss $\left(G^{\prime \prime}\right)$ modulus of Formulation $\mathrm{A}$ and $\mathrm{B}$ at room temperature $\left(25^{\circ} \mathrm{C}\right)$ and body temperature $\left(37^{\circ} \mathrm{C}\right)$ as function of Oscillation stress $(\sigma)$. 
Table 1. Oscillation stress sweep: yield stress and stress flow values of the two tested batches of formulation $\mathrm{A}$ and $\mathrm{B}$ at room temperature $\left(25^{\circ} \mathrm{C}\right)$ and body temperature $\left(37^{\circ} \mathrm{C}\right)$.

\begin{tabular}{llllllll}
\hline & & G$^{\prime}(\mathrm{Pa})$ & $\mathbf{G}^{\prime \prime}(\mathrm{Pa})$ & $\boldsymbol{\sigma}_{\mathrm{y}}(\mathrm{Pa})$ & $\boldsymbol{\sigma}_{\mathrm{f}}(\mathrm{Pa})$ & $\tan \boldsymbol{\delta}$ & $\boldsymbol{\delta}\left(^{\circ}\right)$ \\
\hline Formulation A & Batch A1 & $5.64 \pm 0.004$ & $2.73 \pm 0.002$ & 1.30 & 5.3 & $0.48 \pm 0.001$ & $25.8 \pm 0.05$ \\
$\mathbf{2 5}{ }^{\circ} \mathbf{C}$ & Batch A2 & $5.62 \pm 0.004$ & $2.81 \pm 0.001$ & 1.30 & 5.3 & $0.49 \pm 0.001$ & $26.3 \pm 0.07$ \\
Formulation B & Batch B1 & $0.33 \pm 0.020$ & $0.69 \pm 0.003$ & 0.50 & - & $2.02 \pm 0.019$ & $63.7 \pm 1.08$ \\
$\mathbf{2 5}{ }^{\circ} \mathbf{C}$ & Batch B2 & $0.23 \pm 0.020$ & $0.64 \pm 0.002$ & 0.50 & - & $2.82 \pm 0.020$ & $70.5 \pm 1.14$ \\
Formulation A & Batch A1 & $4.27 \pm 0.002$ & $2.49 \pm 0.001$ & 1.00 & 4.2 & $0.57 \pm 0.001$ & $30.0 \pm 0.03$ \\
$\mathbf{3 7}{ }^{\circ} \mathbf{C}$ & Batch A2 & $4.64 \pm 0.002$ & $2.83 \pm 0.007$ & 1.00 & 4.2 & $0.60 \pm 0.001$ & $30.8 \pm 0.04$ \\
Formulation B & Batch B1 & $0.19 \pm 0.010$ & $0.45 \pm 0.003$ & 0.25 & - & $2.44 \pm 0.021$ & $67.7 \pm 1.20$ \\
$\mathbf{3 7}{ }^{\circ} \mathbf{C}$ & Batch B2 & $0.12 \pm 0.007$ & $0.39 \pm 0.002$ & 0.25 & - & $3.13 \pm 0.020$ & $72.3 \pm 1.14$ \\
\hline
\end{tabular}

elastic modulus decreased, but in a larger proportion (about 43\%) than for formulation A. This is due to the prevalently liquid nature of formulation B. Moreover, measurements showed a lower reproducibility intra-batch and inter-batches (Table 1).

It was possible to estimate the flow stress parameter only with formulation A because only this formulation has a viscoelastic gel-type behaviour for which it was possible to find a crossover point between $G^{\prime}$ and $G^{\prime \prime}$, where the applied stress is capable to convert the behaviour in viscous fluid. In addition, the LVR of formulation $A$ was more than twice higher than that of formulation $B$ both at room and body temperature. Although the yield stress was not particularly high for both suspensions, formulation A retained its gel-like rheological properties in a stress range of solicitations, such as by stirring, in a greater extent than formulation $B$, which instead lost these properties due to its limited elasticity ( $G^{\prime \prime}>$ $\mathrm{G}^{\prime}$ across the $\sigma$ tested range).

\section{Creep recovery}

Figure 2 shows the results of the creep-recovery tests with the two formulations at room $\left(25^{\circ} \mathrm{C}\right)$ and body $\left(37^{\circ} \mathrm{C}\right)$ temperature. With formulation $\mathrm{A}$, the constant stress applied in the first creep phase at $25^{\circ} \mathrm{C}$ was to $1.3 \mathrm{~Pa}$, i.e. the value of $\sigma_{y}$ measured in the oscillation stress sweep test. Similarly, at $37^{\circ} \mathrm{C}$, a constant stress equal to a $\sigma_{\mathrm{y}}$ of $1 \mathrm{~Pa}$ was applied. Importantly, the comparison between the percentages of deformation measured at the two temperatures should take into account the different stress applied, since there are bigger deformations at grater amplitudes of applied stress. Nevertheless, despite at $25^{\circ} \mathrm{C}$ a greater stress was applied, at $37^{\circ} \mathrm{C}$ there was a more consistent deformation, with a peak value at 100 seconds of approximately $\gamma(\%)=600 \%$, vs. $\gamma(\%)=270 \%$ measured at $25^{\circ} \mathrm{C}$. This was due to the temperature effect on the aqueous suspension that determined a greater fluidity and tendency to creep. In both analyses, the induced deformation in the sample during creep was consistent, but in the next phase of recovery it was possible to track down the two elastic and viscose components of deformation: there was an elastic portion of deformation $\gamma_{e}(\%)$, which was recovered from the sample once the stress was removed, thus confirming the viscoelastic

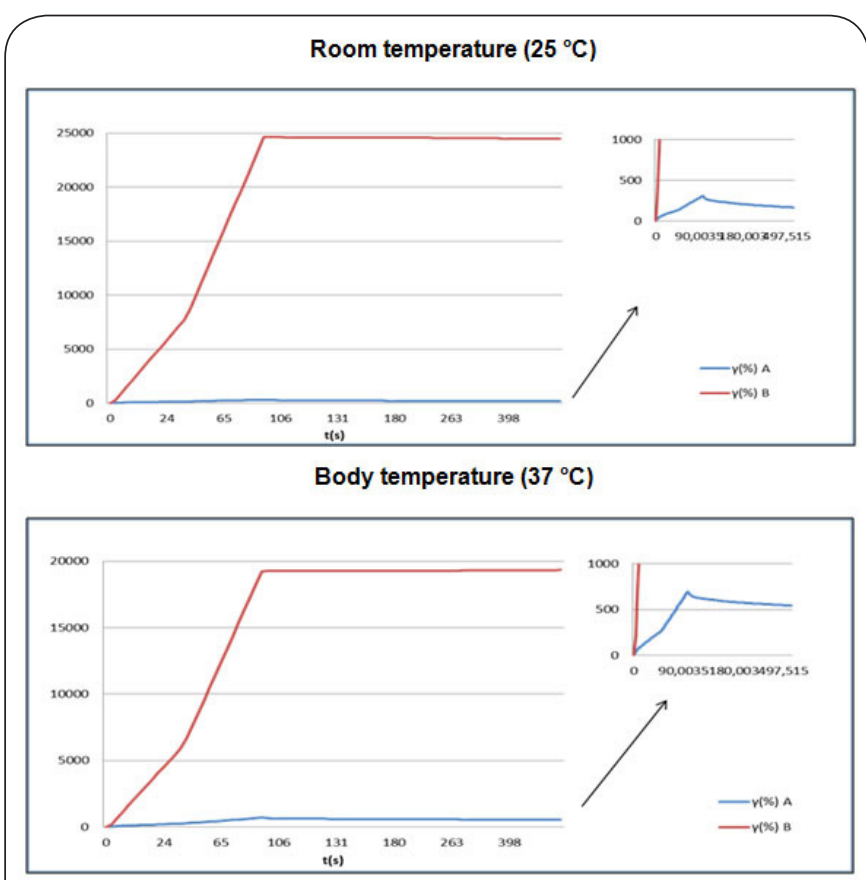

Figure 2. Results of creep-recovery tests. Comparisons of Formulation $\mathbf{A}$ and $\mathbf{B}$ at room temperature $\left(25^{\circ} \mathrm{C}\right)$ and body temperature $\left(37^{\circ} \mathrm{C}\right)$. Applied stress was $1 \mathrm{~Pa}$ for Formulation $\mathrm{A}$ at both temperatures and $0.5 \mathrm{~Pa}\left(25^{\circ} \mathrm{C}\right)$ and $0.25 \mathrm{~Pa}\left(37^{\circ} \mathrm{C}\right)$ for formulation B.

gel-like behaviour observed in the previous test. This elastic deformation was more consistent at room temperature, but remained noticeable even at body temperature.

With regard to formulation $\mathrm{B}$, although the constant stress applied during the creep phase was equal to $0.5 \mathrm{~Pa}$ at $25^{\circ} \mathrm{C}$ and $0.25 \mathrm{~Pa}$ at $37^{\circ} \mathrm{C}$, the percentage of deformation induced in the sample during the creep phase was very high and reached a peak at $100 \mathrm{sec}$ of $\gamma(\%)=23000 \%$ at $25^{\circ} \mathrm{C}$ and of $\gamma(\%)=20000 \%$ at $37^{\circ} \mathrm{C}$, thus indicating the viscose fluid nature of this sample, which largely deformed under the action of an external constant stress. In this case the maximum deformation was observed as a function of the greater stress applied, and 
therefore it was such factor to drive the extent of the measured deformation and not the temperature effect. The liquid and viscose nature of formulation B was further confirmed by the total absence of elastic portion of the deformation, i.e. in the recovery phase there was only the viscous component. Once the stress was removed the sample did not recover the deformation that remained irreversible.

Assuming that the applied stress during the creep phase had different values as they were calibrated on the value of yield stress of each sample, it is evident that both suspensions underwent important deformations, but the deformation of formulation $B$ was twice as higher as that of sample $A$, despite the stress applied on $B$ at the two temperatures was always less than the half of that applied on formulation A. Moreover, formulation $A$ partly recovered the deformation, maintaining an elastic strain portion also at $37^{\circ} \mathrm{C}$, while formulation $\mathrm{B} \mathrm{com}$ pletely and irreversibly dissipated the deforming stress energy, for which the B elastic component was void, and the viscous portion coincided with the total percentage of deformation.

\section{Frequency sweep}

Figure 3 shows the results of the frequency sweep tests with the two suspensions at room $\left(25^{\circ} \mathrm{C}\right)$ and body $\left(37^{\circ} \mathrm{C}\right)$ temperature. Once again, the elastic modulus $\mathrm{G}^{\prime}$ was higher than the viscous modulus G", i.e. was index of a gel-like viscoelastic behaviour. Notably, the crossover point of the modules shifted to higher frequencies as the temperature increased. The reciprocal of the frequency (expressed in $\mathrm{Hz}$ ) at which occurred $\mathrm{G}^{\prime}=\mathrm{G}^{\prime \prime}$

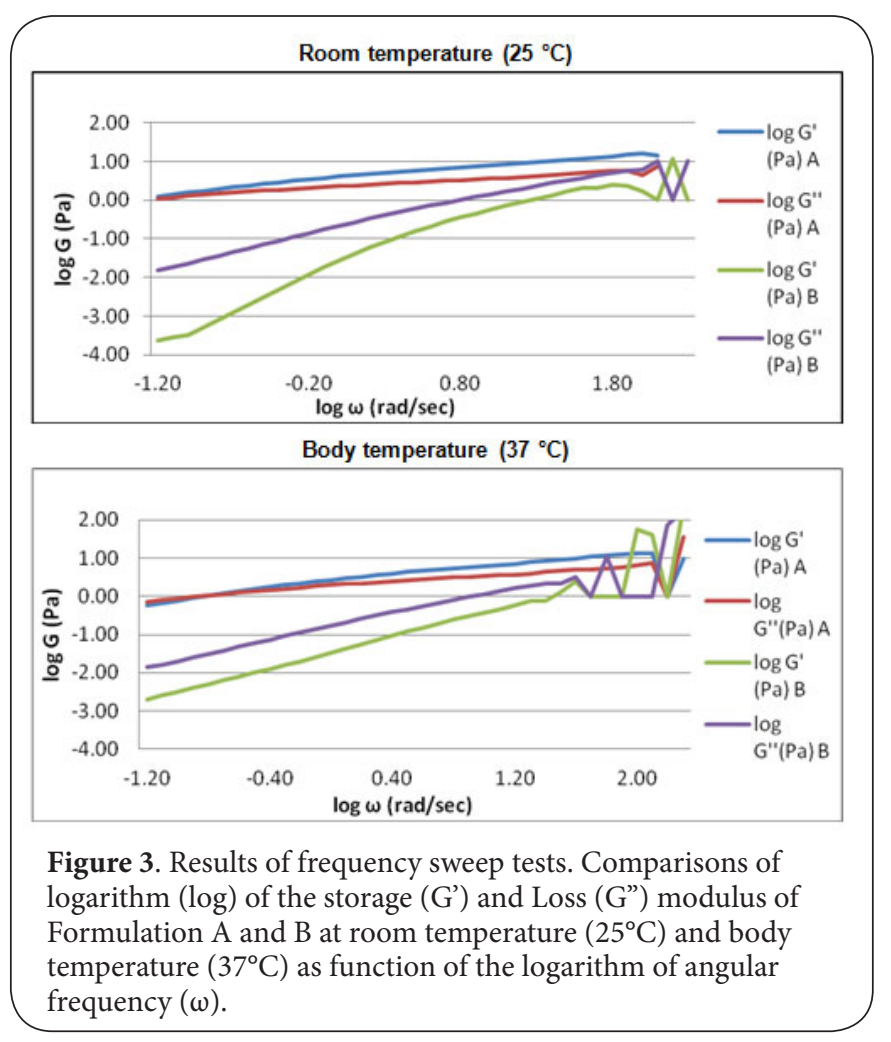

represents the relaxation time of the chemical and physical interactions that develop inside of the suspension [18]. At $25^{\circ} \mathrm{C}$, the crossover point was $0.01 \mathrm{~Hz}$ and the relaxation time was 100 seconds. At $37^{\circ} \mathrm{C}$, the crossover point was $0.025 \mathrm{~Hz}$ and the relaxation time was 40 seconds. These findings confirmed the viscoelastic behaviour attributable to a viscous fluid for formulation $B$, with $G^{\prime \prime}>G^{\prime}$ across the whole frequency range and at both temperatures.

Generally, the slope of the curve $\log \left(G^{\prime}\right) \mathrm{vs} \cdot \log (\omega)$ is the parameter observed as an index of the tendency to settle and to present phase separation. Figure 3 shows that the curves with increased slope were those recorded for the formulation $B$, which is therefore more subject to instability phenomena compared to formulation A. It was also possible to estimate the concentration degree of the formulation: in fact, in diluted suspension viscous properties prevailed over the elastic ones and the $\mathrm{G}^{\prime \prime}$ was always greater than $\mathrm{G}^{\prime}$, even if the two modules tended to get closer at high frequencies as observed for formulation B. On the contrary, in formulation A the $G^{\prime \prime}$ and $G^{\prime}$ modules intersected each other. This behaviour takes place in case of concentrated solutions, because, at high frequencies, the tendency to an elastic behaviour prevails.

\section{Viscosimetry test}

At $25^{\circ} \mathrm{C}$, formulation A showed the characteristic flow curve of a 'shear thinning' pseudoplastic fluid, with a flow threshold. The calculation of this parameter was carried out using the software 'Rheosoft' that allows a 'Best fitting' elaboration in order to identify the mathematical model able to best interpolate the curve. Among those proposed, the'HerschellBulkley' model (with greater confidence level) was used, and identified $\sigma_{\mathrm{y}}=1.26 \mathrm{~Pa}$, a value consistent with that found in the oscillation stress sweep test. Similarly, at $37^{\circ} \mathrm{C}$ the same model identified $\sigma_{y}=1.3 \mathrm{~Pa}$, again consistent with findings of the oscillation stress sweep test. The flow curves recorded for formulation B showed the typical trend recorded for "shear thinning" pseudo plastic fluids.

Figure 4 shows the viscosity curves profile of the two suspensions at room $\left(25^{\circ} \mathrm{C}\right)$ and body $\left(37^{\circ} \mathrm{C}\right)$ temperature. Table 2 shows the values of viscosity.

The log $\eta$ vs log $(\gamma)$ trend, which is characteristic of pseudoplastic fluids, was clearly visible both in the first Newtonian region of the curve and in the shear-thinning region. By elaborating the experimental curves via a 'Rheosoft' model, it was possible to obtain the interpolation through mathematical model functions in a way that extrapolated the values of the parameter 'zero-rate viscosity' $\left(\eta_{0}\right)$ and 'infinity-rate viscosity' $\left(\eta_{\infty}\right)$. For both curves, the interpolation function that better fitted the experimental data was the Carreau-Yasuda model although the value of viscosity at Infinitum obtained for Form, $B$ was not realistic (negative) and so it is not reported, the calculated parameters are summarized in Table 2. As expected, with increasing temperature, a decrease in suspensions viscosity occurred as a result of increased fluidity, tendency to 

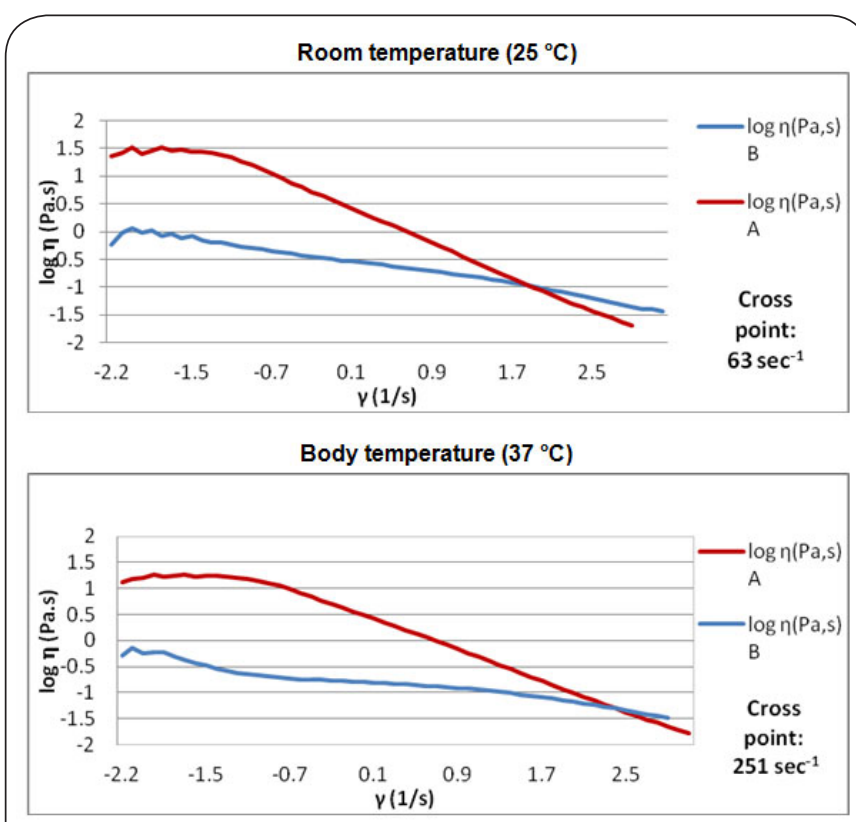

Figure 4. Viscosity curves profile of Formulation A and B at room temperature $\left(25^{\circ} \mathrm{C}\right)$ and body temperature $\left(37^{\circ} \mathrm{C}\right)$ : Logarithm of Viscosity $(\eta)$ as function of of shear rate $(\gamma)$.

Table 2. Results of viscosity tests: values of Formulation A and $B$ at room temperature $\left(25^{\circ} \mathrm{C}\right)$ and body temperature $\left(37^{\circ} \mathrm{C}\right)$.

\begin{tabular}{lcll}
\hline & $\eta^{\circ} \infty$ (Pa.s) & $\eta \infty($ Pa.s $)$ & $\eta 500($ Pa.s $)$ \\
\hline Formulation A $25^{\circ} \mathrm{C}$ & 27.960 & 0.004 & 0.03 \\
Formulation B 25 $5^{\circ} \mathrm{C}$ & 0.393 & N.C. & 0.06 \\
Formulation A $37^{\circ} \mathrm{C}$ & 18.015 & 0.001 & 0.05 \\
Formulation B 37 $\mathrm{C}$ & 0.185 & N.C. & 0.02 \\
\hline
\end{tabular}

flow and decreased viscosity.

In contrast with previous findings, the first Newtonian region of the curve was much less attributable to the ideal-theoretical trend that would be expected for "shear thinning" fluids, and this was due to the fact that measurements collected at low $\gamma$ have poor reproducibility both intra-batch and interbatches, and to the viscous fluid nature of this suspension, which is characterized by small values of viscosity and was therefore difficult to be measured especially at low $\gamma$ (need for appropriate time delay for the system to balance). From the values reported in Table 2 it is evident that, at the two different temperatures, formulation $A$ had a viscosity at rest twice as higher as that of formulation B. As mentioned earlier the $\eta_{\infty}$ value, extrapolated for the suspension $B$ sample through fitting, gave negative values, probably due to the lack of confidence on the mathematical model used, since the experimental curve differed greatly from the ideal trend of pseudoplastic fluids. To remedy this inconsistency, it was decided to tabulate the experimental viscosity recorded at the maximum value of shear rate imposed to the system. In such range of $\gamma$, suspension $B$ had a higher viscosity than suspension $A$. This is an indication of the fact that the latter sample, although showing higher viscosity at rest, begins to slide when subjected to large flow gradients and its viscosity decreases consistently up to being even lower than that of the fluid-viscose suspension B. Figure 4 shows the moment at which this trend inversion between the two samples was recorded. Flow gradients so high to determine a consistent viscosity fall for A can be reached during shaking of the bottle or during the squeezing process involved in the application of the pharmaceutical product (see supplementary materials). Therefore, consistently with what was observed during the test with the products performance, it can be derived that, at rest, formulation $A$ has a viscosity significantly higher than that of formulation $B$ in the therapeutic site, thus remaining in place for a longer time.

\section{Time sweep}

Figure 5 shows the results of the time sweep tests of the two suspensions at room $\left(25^{\circ} \mathrm{C}\right)$ temperature. When subjected to stresses of constant amplitude and frequency, both suspensions recovered their rheological properties in a time interval of about 10 seconds for formulation $A$ and 16 seconds for formulation B. Both formulations showed an increase in their structural consistency, probably due to an increase in the number of physical interactions which developed in the suspension [19].

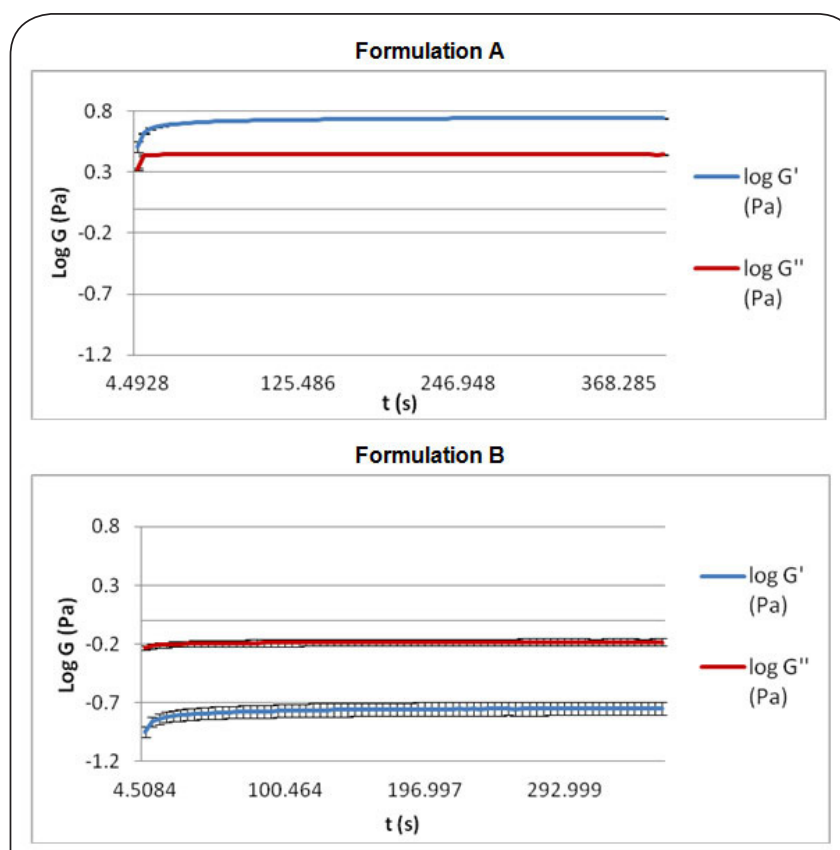

Figure 5. Results of the time sweep tests with the two formulations $\mathrm{A}$ and $\mathrm{B}$ at room $\left(25^{\circ} \mathrm{C}\right)$ temperature: Plot of the Logarithm of storage ( $\left.G^{\prime}\right)$ and Loss $\left(G^{\prime \prime}\right)$ modulus as function of time while keeping stress and frequency at constant values $(0,5 \mathrm{~Pa}-0,5 \mathrm{~Hz})$. 


\section{Temperature sweep}

Figure 6 shows the results of the temperature sweep tests of the two formulations. As the temperature increased, both samples showed a decrease of the elastic modulus, as a consequence of the increase of the system fluidity. In the interval between $5^{\circ} \mathrm{C}$ and $30^{\circ} \mathrm{C}$, the elastic modulus $\mathrm{G}^{\prime}$ slowly decreased for formulation $A$, with a flatter slope compared to that of formulation $B$, which is an index of a stronger tendency to maintain the elastic properties during heating. In contract, the cooling ramp between $25^{*} \mathrm{C}$ and $5^{\circ} \mathrm{C}$ showed an increase of the elastic modulus $G^{\prime}$ for formulation $B$, which is indicative of a greater structuration with decreasing temperature, a phenomenon that was not observed for formulation $A$.

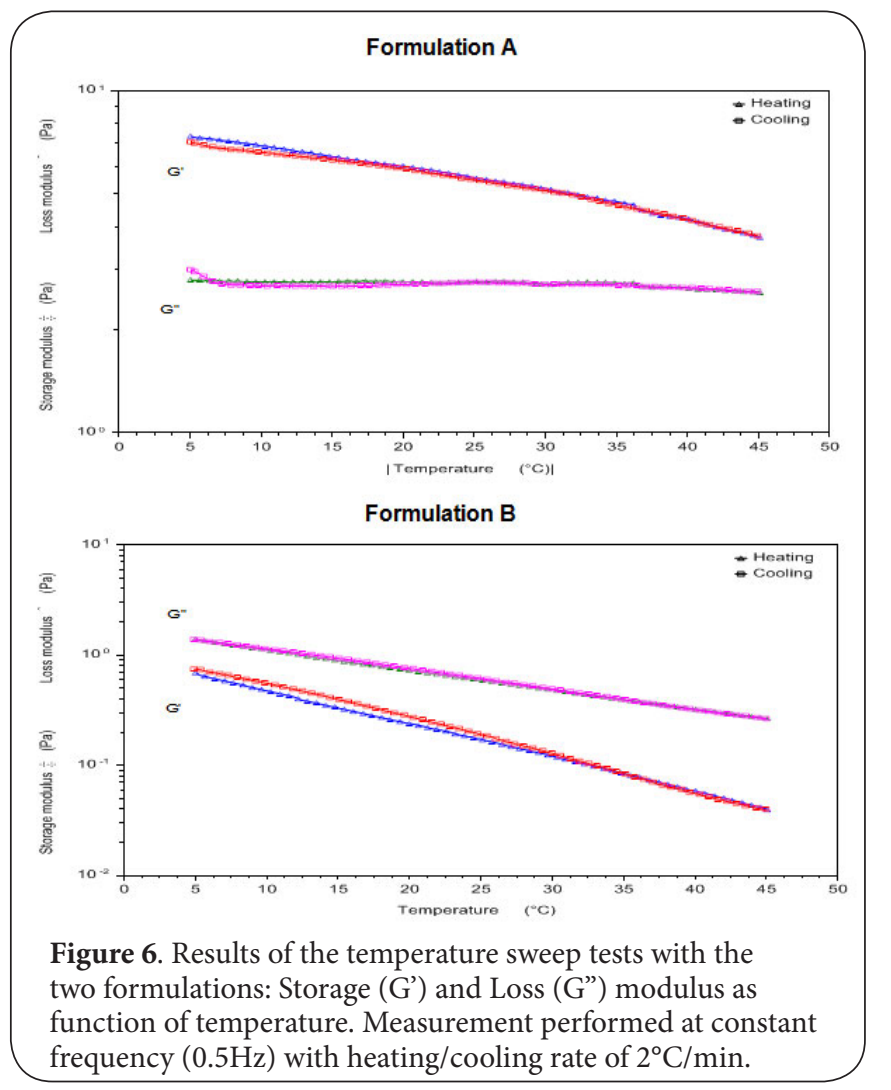

\section{Discussion}

The tests herein described were performed to determine a complete rheological characterization of two marketed BDP aqueous suspension formulations for topical mucosal application used in the treatment of IBD (CD and UC). The viscoelastic behaviour of the two formulations was defined by means of rheological tests aimed at identifying differences in structural consistency between the two tested formulations. The rheological characterization of the two aqueous suspensions was evaluated on two different batches for each formulation by means of a preliminary oscillation stress sweep test aimed at evaluating the linear viscoelastic range and therefore the values of yield stress for each sample. This approach allows predicting whether differences between the rheological properties of the two formulations may be associated with differences in effectiveness and performance in clinical use.

The results of the battery of tests have highlighted important differences between the two formulations. First, the viscous and elastic $G^{\prime}$ and $G^{\prime \prime}$ modules calculated in the oscillatory tests have allowed to define the viscoelastic behaviour of formulation $A$ as a gel-like type, in which the elastic properties dominate over the viscous-fluid ones and therefore the stress energy is stored in the form of reversible deformation [20]. Conversely, the viscoelastic behaviour of formulation B were of viscous-fluid type, as the viscous properties prevailed over the elastic one and therefore the energy of the stress was completely dissipated in the form of irreversible deformation [18]. Furthermore, the oscillation stress sweep test showed that formulation $A$ retains its rheological properties in a range of applied stress (LVR) in an extent that was almost twice as higher as that of formulation B. This stress can be assimilated to the agitation stage of the bottle prior to the drug application, which acts as an external stress. This is of importance considering that the bottle agitation before the application precedes the application of the product on the mucosal tissue, and thus the suspension is subjected to an external stress before usage.

The results of the creep-recovery test confirmed a liquidlike behaviour for formulation $B$ and a gel-like behaviour for formulation $A$, which was able to recompose the deformation once the stress ceased. Findings have indicated that that both formulations undergo high strains following an external stress. However, while formulation A was able to recover an elastic portion of this deformation and partially restore its original structure, formulation B remained completely deformed and dissipated all the energy in the form of irreversible deformation. This test can be assimilated to the application phase involved in the 'squeezing' of the bottle (creep stage) and the positioning of the suspension at the mucosal therapeutic site. In this scenario both formulations flow out of the container and reach easily the application site (as their deformation and tendency to flow were high), but once the stress is ceased, formulation $B$ is completely deformed as viscous liquid and its tendency to flow remains unchanged. On the contrary, formulation A partly reconstitutes itself and maintains a gel-like component able to remain longer at the therapeutic site of action.

It has been experimentally demonstrated that the frequency sweep test is often related to the stability of the suspension [19]. With this respect, the results of this test showed a more pronounced 'structural integrity' for formulation A than for formulation B. The results of viscosimetry tests have shown that formulation $A$ has a viscosity at rest twice as higher as that of formulation B. Therefore, once it is placed at the site of action (at rest), it is expected that formulation $A$, having a much high viscosity, remains localized at the target site for a prolonged time determining a long-lasting effect. Moreover, 
the administration of formulation $\mathrm{A}$ is also facilitated by a more favourable sliding, considering the lower viscosity at high shear of product application. This behaviour is consistent with findings in the creep-recovery test: after the stress application, only formulation A was able to recover the elastic train portion recomposing at rest part of its original structure, whereas formulation $B$ totally lost its shape and did not recover from the deformation at all. Both formulations showed high recovery capacity of the viscoelastic properties as a function of time. In fact, both formulations required very short time intervals, which were shorter than 20 seconds. As the temperature increased, both formulation $A$ and $B$ showed a reduction of the elastic component, as it can be expected from aqueous-based suspensions, whose fluidity increases with temperature.

The different behaviour of the two formulations can be ascribed to the thickener agents (Xanthan gum and Na carboxymethyl cellulose) used and likely to their concentration, but influence due to emulsifier used in the formulation $A$ (e.g. cetostearyl alcohol) can not be excluded. The differences in rheological properties between the two BDP tested formulations may have important implications in their use in clinical practice. Considering that the two formulations are pharmaceutical suspensions for topical and mucosal application, it can be hypothesized that a higher viscosity, a gel-like behaviour and pronounced elastic properties may determine a longer time of drug permanence in the site of action. This will reduce the ease of the product's ejection by patients and increases the exposure time of the affected surface to the therapeutic action. A consequent improvement of adherence to treatment may also be expected, as patients do not require further administration, and hence the overall therapeutic efficacy may be maximized with reduced risks of adverse effects. In fact, a key issue with topical intra-rectal therapy in clinical practice is the retention time of enema solutions, which is significantly reduced in the presence of rectal inflammation [21]. Therefore, it is likely that a more viscous solution may remain in contact with the affected mucosa for a longer time than non-viscous solutions, thus maximizing drug effectiveness.

\section{Conclusions}

The overall results of the tests performed to define a complete rheological characterization of two marketed BDP aqueous suspension formulations for topical mucosal application, have shown that formulation $A$ (has more performing rheological properties than formulation B (. Furthermore, formulation A has a viscoelastic gel-like behaviour compared to viscous-fluid characteristics of formulation B. These properties may lead to important differences in the performance of the two tested formulations in clinical practice. However, such potential differences should be demonstrated in randomized clinical trials that include adequate patients' sizes and using appropriate outcome measures for a reliable assessment of efficacy and safety.

\section{Competing interests}

The authors declare that they have no competing interests.

\section{Authors' contributions}

\begin{tabular}{|l|c|c|c|c|c|}
\hline Authors' contributions & AD & FS & GT & LP & MV \\
\hline Research concept and design & $\checkmark$ & $\checkmark$ & -- & -- & -- \\
\hline Collection and/or assembly of data & $\checkmark$ & -- & -- & -- & -- \\
\hline Data analysis and interpretation & $\checkmark$ & $\checkmark$ & -- & -- & -- \\
\hline Writing the article & $\checkmark$ & $\checkmark$ & $\checkmark$ & $\checkmark$ & $\checkmark$ \\
\hline Critical revision of the article & -- & $\checkmark$ & $\checkmark$ & $\checkmark$ & $\checkmark$ \\
\hline Final approval of article & -- & -- & -- & -- & -- \\
\hline Statistical analysis & -- & -- & -- & -- & -- \\
\hline
\end{tabular}

\section{Acknowledgments and fundings}

The authors wish to acknowledge prof Gianfilippo Palmieri (University of Camerino) for kindly review the manuscript and Dr Erica Verdini that has completed some of the works reported in the supporting Information. This research did not receive any specific grant from funding agencies in the public, commercial, or not-for-profit sectors.

\section{Publication history}

Editor: Anchalee Jintapattanakit, Mahidol University, Thailand Received: 05-Apr-2018 Final Revised: 05-Jun-2018

Accepted: 08-Jun-2018 Published: 24-Jun-2018

\section{References}

1. Maccioni F, Viola F, Carrozzo F, Di Nardo G, Pino AR, Staltari I, Al Ansari N, Vestri A, Signore A, Marini M and Cucchiara S. Differences in the location and activity of intestinal Crohn's disease lesions between adult and paediatric patients detected with MRI. Eur Radiol. 2012; 22:2465-77. | Article I PubMed

2. Riegler G, Tartaglione MT, Carratu R, D'Inca R, Valpiani D, Russo MI, Papi C, Fiorentini MT, Ingrosso M, Andreoli A and Vecchi M. Age-related clinical severity at diagnosis in $\mathbf{1 7 0 5}$ patients with ulcerative colitis: a study by GISC (Italian Colon-Rectum Study Group). Dig Dis Sci. 2000; 45:462-5. | PubMed

3. Baumgart DC and Sandborn WJ. Inflammatory bowel disease: clinical aspects and established and evolving therapies. Lancet. 2007; 369:164157. | Article | PubMed

4. Duricova D, Burisch J, Jess T, Gower-Rousseau C and Lakatos PL. Agerelated differences in presentation and course of inflammatory bowel disease: an update on the population-based literature. J Crohns Colitis. 2014; 8:1351-61. I Article I PubMed

5. Baid SK and Nieman LK. Therapeutic doses of glucocorticoids: implications for oral medicine. Oral Dis. 2006; 12:436-42. | Article | PubMed

6. Truelove SC and Hambling $\mathrm{MH}$. Treatment of ulcerative colitis with local hydrocortisone hemisuccinate sodium; a report on a controlled therapeutic trial. Br Med J. 1958; 2:1072-7. | PubMed Abstract | PubMed FullText

7. Truelove SC. Systemic and local corticosteroid therapy in ulcerative colitis. Br Med J. 1960; 1:464-7. | PubMed Abstract | PubMed FullText

8. McDougal L. Treatment of ulcerative colitis with rectal steroids. Lancet. $1963 ; 1: 826-827$.

9. Lee DA, Taylor M, James VH and Walker G. Rectally administered prednisolone--evidence for a predominantly local action. Gut. 1980; 21:215-8. | Article | PubMed Abstract | PubMed FullText

10. Harris DM. Some properties of beclomethasone dipropionate and related steroids in man. Postgrad Med J. 1975; 51 Suppl 4:20-5. | PubMed

11. Nunes T, Barreiro-de Acosta M, Marin-Jimenez I, Nos P and Sans M. Oral 
locally active steroids in inflammatory bowel disease. J Crohns Colitis. 2013; 7:183-91. | Article | PubMed

12. De Cassan C, Fiorino G and Danese S. Second-generation corticosteroids for the treatment of Crohn's disease and ulcerative colitis: more effective and less side effects? Dig Dis. 2012; 30:368-75. | Article | PubMed

13. Brunner M, Ziegler S, Di Stefano AF, Dehghanyar P, Kletter K, Tschurlovits M, Villa R, Bozzella R, Celasco G, Moro L, Rusca A, Dudczak R and Muller M. Gastrointestinal transit, release and plasma pharmacokinetics of a new oral budesonide formulation. Br J Clin Pharmacol. 2006; 61:31-8. | Article | PubMed Abstract | PubMed FullText

14. Van Assche G, Manguso F, Zibellini M, Cabriada Nuno JL, Goldis A, Tkachenko E, Varoli G, Kleczkowski D, Annese V, D'Heygere F and Balzano A. Oral prolonged release beclomethasone dipropionate and prednisone in the treatment of active ulcerative colitis: results from a double-blind, randomized, parallel group study. Am J Gastroenterol. 2015; 110:708-15. I Article I PubMed

15. Manguso F, Bennato R, Lombardi G, Riccio E, Costantino G and Fries W. Efficacy and Safety of Oral Beclomethasone Dipropionate in Ulcerative Colitis: A Systematic Review and Meta-Analysis. PLoS One. 2016; 11:e0166455. | Article | PubMed Abstract | PubMed FullText

16. Kumana CR, Seaton T, Meghji M, Castelli M, Benson R and Sivakumaran $T$. Beclomethasone dipropionate enemas for treating inflammatory bowel disease without producing Cushing's syndrome or hypothalamic pituitary adrenal suppression. Lancet. 1982; 1:579-83. | Article | PubMed

17. Shaw MT. Introduction to Polymer Rheology. Experimental Methods. In Montgomery T. Shaw (Eds.), Introduction to Polymer Rheology, Wiley 2012; 125-149.

18. Bonacucina G, Martelli S and Palmieri GF. Rheological, mucoadhesive and release properties of Carbopol gels in hydrophilic cosolvents. Int J Pharm. 2004; 282:115-30. I Article I PubMed

19. Mezger TG. The Reology Handbook. $3^{\text {rd }}$ Revised Edition, Hanover: Vincentz Network, 2011; Chapter 8.4.4: 171-174.

20. Tamburic $S$ and Craig DQM. Rheological of polyacrylic acid hydrogels. Pharm Sci. 1995; 1:107-109.

21. Ingram JR, Rhodes J, Evans BK, Newcombe RG and Thomas GA. Comparative study of enema retention and preference in ulcerative colitis. Postgrad Med J. 2005; 81:594-8. | Article | PubMed Abstract | PubMed FullText

\section{Citation:}

Demma AV, Sartor F, Tontini GE, Pastorelli L and Vecchi M. Rheological characterization of two different suspension formulations of beclomethasone dipropionate enemas for rectal administration.

J Pharm Technol Drug Res. 2018; 7:1.

http://dx.doi.org/10.7243/2050-120X-7-1 